Progress on the Development of a Single Line of Sight X-ray Framing Camera

J.P. Holder, D.K. Bradley, C.M. Damian, K.W. Piston, P.M. Bell, A.K.L. Dymoke-Bradshaw, J.D. Hares

April 16, 2004

15th Topical Conference on High Temperature Plasma Diagnostics San Diego, CA, United States April 19, 2004 through April 22, 2004 
This document was prepared as an account of work sponsored by an agency of the United States Government. Neither the United States Government nor the University of California nor any of their employees, makes any warranty, express or implied, or assumes any legal liability or responsibility for the accuracy, completeness, or usefulness of any information, apparatus, product, or process disclosed, or represents that its use would not infringe privately owned rights. Reference herein to any specific commercial product, process, or service by trade name, trademark, manufacturer, or otherwise, does not necessarily constitute or imply its endorsement, recommendation, or favoring by the United States Government or the University of California. The views and opinions of authors expressed herein do not necessarily state or reflect those of the United States Government or the University of California, and shall not be used for advertising or product endorsement purposes. 


\title{
Progress on the Development of a Single Line of Sight X-ray Framing Camera
}

D.K. Bradley, J.P. Holder, C.M. Damian, K. W. Piston and P.M. Bell

Lawrence Livermore National Laboratory

A. K. L. Dymoke-Bradshaw, and J. D. Hares

Kentech Instruments Ltd.

\begin{abstract}
High-speed micro-strip micro-channel plate (MCP) x-ray framing cameras are a well established diagnostic for laser plasma experiments. Each frame acquired with these devices requires a separate image, and with most reasonable x-ray optics, a separate line of sight, causing potential parallax problems. Gated image tubes have a single line of sight capability, but the conventional designs have not been effectively extended to the short gating times of the micro-strip-line MCP camera. A hybrid camera combining image tube and micro-strip-line MCP technology has been under development at LLNL in collaboration with UR/LLE, and KENTECH Instruments. The key feature of this single line of sight (SLOS) hybrid image tube is a deflection assembly that continuously divides the electrons from a single photocathode $\mathrm{x}$-ray image into a set of four electron images. Temporal gating of these images is carried out using a microstripline microchannel plate framing camera module positioned at the image plane of the electron tube. Characterization measurements performed using both X-rays from a Manson source and from laser generated plasmas, will be presented. Some implementation improvements will be discussed. The results will be compared to simulations carried out using the charged particle optics code SIMION. Various dissector designs were simulated in an effort to improve the image quality of the system.
\end{abstract}




\section{Introduction}

A hybrid camera combining image tube and micro-strip-line MCP technology has been under development at Lawrence Livermore National Lab (LLNL) in collaboration with University of Rochester Laboratory for Laser Energetics (UR/LLE , and KENTECH Instruments $[1,2,3]$. This camera uses the well established high-speed microchannel plate (MCP) $\mathrm{x}$-ray framing camera technology that is typically used to detect soft x-rays. In these devices, each frame acquired requires a separate image, and with most reasonable X-ray optics, a separate line of sight[4]. These multiple lines of sight can cause problems in performing or interpreting some experiments. Image tubes (streak camera tubes used in multi-framing mode) have a single line of sight capability, but the conventional designs, typically with stair step defection voltages, have not been effectively extended to the short gating times of the strip-line MCP camera. To have an alternative solution to this problem we use image tube technology to produce an x-ray beam splitter to produce the multiple images for the strip-line MCP framing camera. This option may allow a single $\mathrm{x}$-ray optic to be optimized for a single line of sight. With appropriate materials and construction techniques this hybrid camera can also provide some protection against hard x-ray background signals since the electrons are deflected away from the optical line of sight of the camera.

The key feature of this single line of sight (SLOS) hybrid framing camera's image tube is a deflection assembly that continuously divides the electrons from a single photocathode x-ray image into a set of four electron images. The electrostatic plates of this "dissector" act as a set of four deflection prisms (in the present implementation a stacked pair of biprisms) in the Fourier plane (cross over) of the image tube, directing the electrons to 
different images on the basis of their ejection angles from the photocathode (radial velocity spectrum). Temporal gating of these images is carried out using a standard gated micro-channel plate (MAX) module positioned at the image plane of the electron tube, with the micro-strips arranged so that the images can be gated at different times. A simple diagram of the SLOS camera configuration is shown in Figure 1.

\section{Previous Results}

The single line of sight camera system has been tested with CW Manson x-ray sources, with a short pulse $200 \mathrm{~nm}$ laser (Bechtel-Nevada Livermore facility) [ 1] and with laser produced X-rays (Janus laser facility)[2 ]. Baseline results were reported in reference [1]. In these results the fundamental timing and imaging capabilities of the camera were found to be promising, with 240 ps gating and with an MTF slightly worse than the MCP alone, but similar to other MCP intensified streak camera systems. Plasma x-rays from gold foils irradiated by the Janus laser were used to illuminate resolution grids placed over the SLOS photocathode. Poorly focused images resulted. Moving the camera from a Manson source to Janus seemed to cause system degradation from transportation induced instabilities. In later Janus experiments, power supply variations were found that seem to be the root cause of these focusing problems. With power supply readjustments, the gated and ungated images on Janus experiments are consistent to the previous results on the short pulse laser and CW x-ray sources. An additional Janus experimental series confirmed these problems. These experiments were reported in ref [2].

\section{New Results Using DC X-ray Sources}

The power supply problem has been solved by the construction of an alternate power supply system. Since the development of the SLOS camera seems to require both 
stability and fine adjustability single high voltage power supply and voltage divider previously used was replaced with a system containing four precision high voltage (0$15 \mathrm{kV}$ ) power supplies. Each of these power supplies are proportionally controlled by dial indicated 10 turn potentiometers. These low voltage potentiometers are between fixed resistors that restricting the range of operation of each supply with values appropriate for the intended electron optics element (i.e. Photocathode, Mesh, Focus, and Zoom).

The adjustability, stability and robustness of the new power supply system allowed the acquisition of the image shown in Figure 2. With this level of control, we can see aberrations in the separate electron images that are due to variations in shapes of the dissector plates, most likely due to warping due to the heat of soldering the plates to their carrier circuit board. Figure 3 is a pair of horizontal line-outs from Figure 2, the left plot is from the lower left dissected image and the right plot is from the thru X-ray image. For the electron image the slit spacing shows that the electron optics magnification is $\sim 1.11$, so the magnified $100 \mu \mathrm{m}$ slit width should be $111 \mu \mathrm{m}$. A Gaussian PSF with a FWHM of $129 \mu \mathrm{m}$ convolved with such an effective slit gives the observed width of 152 $\mu \mathrm{m}$. This corresponds to a photocathode point spread function with a FWHM of $116 \mu \mathrm{m}$. As a check, we also measure the "thru x-ray" image, which has a point projection magnification of 1.57 due to the geometry of the experiment. The observed $193 \mu \mathrm{m}$ linewidths can be modeled by convolving the 1.57 magnified $100 \mu \mathrm{m}$ slit, a FWHM Gaussian MCP PSF of $50 \mu \mathrm{m}$ and the "pinhole camera" 0.57 de-magnified image of the $\sim 250 \mu \mathrm{m}$ FWHM Gaussian size of the Manson x-ray source. Similar results were obtained for vertical line-outs, indicating that a good electron optics focus has been found. 
We have improved the methods used to construct the dissector assembly. A fixture for clamping and holding the deflection electrodes nearly along their whole length has been constructed and used to assemble a set of plates with thicker outer deflection plates to additionally minimize the chance of electrode warping. A completed assembly can be seen in Figure 4. For this test case only one thickness of circuit board was used though we plan to test a double thickness of printed circuit board (i.e. double spacing of bi-prism pairs) to confirm some simulations previously reported.

When this set was able to be tested the fiber coupled CCD camera that we had been using was no longer available for our use. A preliminary image (Figure 5) was taken using a lens coupled CCD camera. Note the focus settings were reset using values that were determined several months earlier. Also the x-ray source was operated at maximum current which was previous observed to broaden the much more strongly source size dependent thru $\mathrm{x}$-ray image. The inter-image balance is not optimally set but, as shown in Figure 6, the grid rectangle "point" images are more self similar than in images with the previous electron bi-prism construction

\section{Simulation and Alternate Configurations}

An effort is underway to improve the image quality of the SLOS camera through experiment and modeling. The electron optics of the camera is modeled using the charged particle optics code SIMION[5]. An external program is used to generate starting electron trajectories for SIMION thatsample the angular and energy distributions of an x-ray photocathode[2,6]. At present non-interacting 'electrons' are simulated. The SIMION simulation results are used to create synthetic images that can be analyzed in the same way as experimental images. Small modifications to the dissector configuration 
(presently equal size parallel plates are used) seem to improve the camera imaging performance in these simulations. When testing these small variations on the experimental systems, the aberrations due to dissector construction problems were observed.

\section{Conclusions and Outlook}

Further tests on better fabricated dissectors of the double bi-prism type will continue using a Manson x-ray source then on pulsed x-ray sources. Additionally, a coplanar, 'quad-prism' dissector system has been simulated. This system, which might be built using thin film construction techniques, has the promise of improved image resolution and better intra-image intensity balance. If good performance of the SLOS camera system is achieved, then the TIM air box packaging of the system for LLE/Omega or LLNL/NIF deployment can proceed. This system must also compete with and adapt to improvements in solid state $\mathrm{x}$-ray camera gating technology development[7].

This work was performed under the auspices of the U.S. Department of Energy by University of California Lawrence Livermore National Laboratory under Contract No. W-7405-ENG-48.

[1] D.K. Bradley, P.M. Bell, A.K.L. Dymoke-Bradshaw , J.D. Hares, R.E. Bahr,V.A. Smalyuk, D.R. Hargrove and K. Piston, Rev. Sci. Instrum. 72, 694(2001)

[2] J.P. Holder, K.W. Piston, D.K. Bradley, P.M. Bell, A.K.L. Dymoke-Bradshaw and J.D. Hares, Rev. Sci. Instrum. 74, 2191(2003)

[3]KENTECH Instruments, www.kentech.co.uk 
[4]J. D. Kilkenny, Laser Part. Beams 9, 49 (1991).

[5] D. A. Dahl, SIMION three-dimensional version 7.0 User's Manual, INEL-95/0403 (2000).

[6] B. L. Henke, J. A. Smith, and D. T. Attwood, J. Appl. Phys. 48, 1852 (1977).

[7]R.K. Reich et. al. ,Rev Sci. Instrum. 74, 2027(2003).

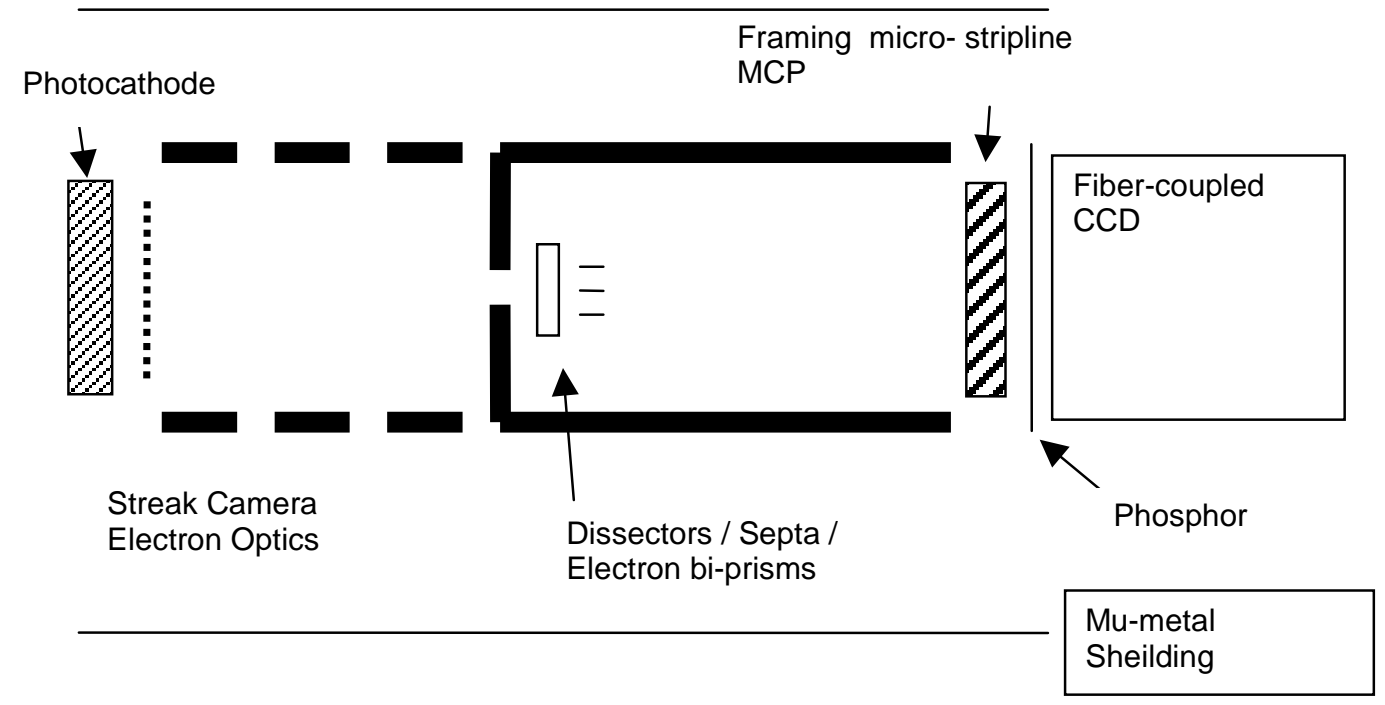


Figure 1 Simplified Schematic of SLOS Camera

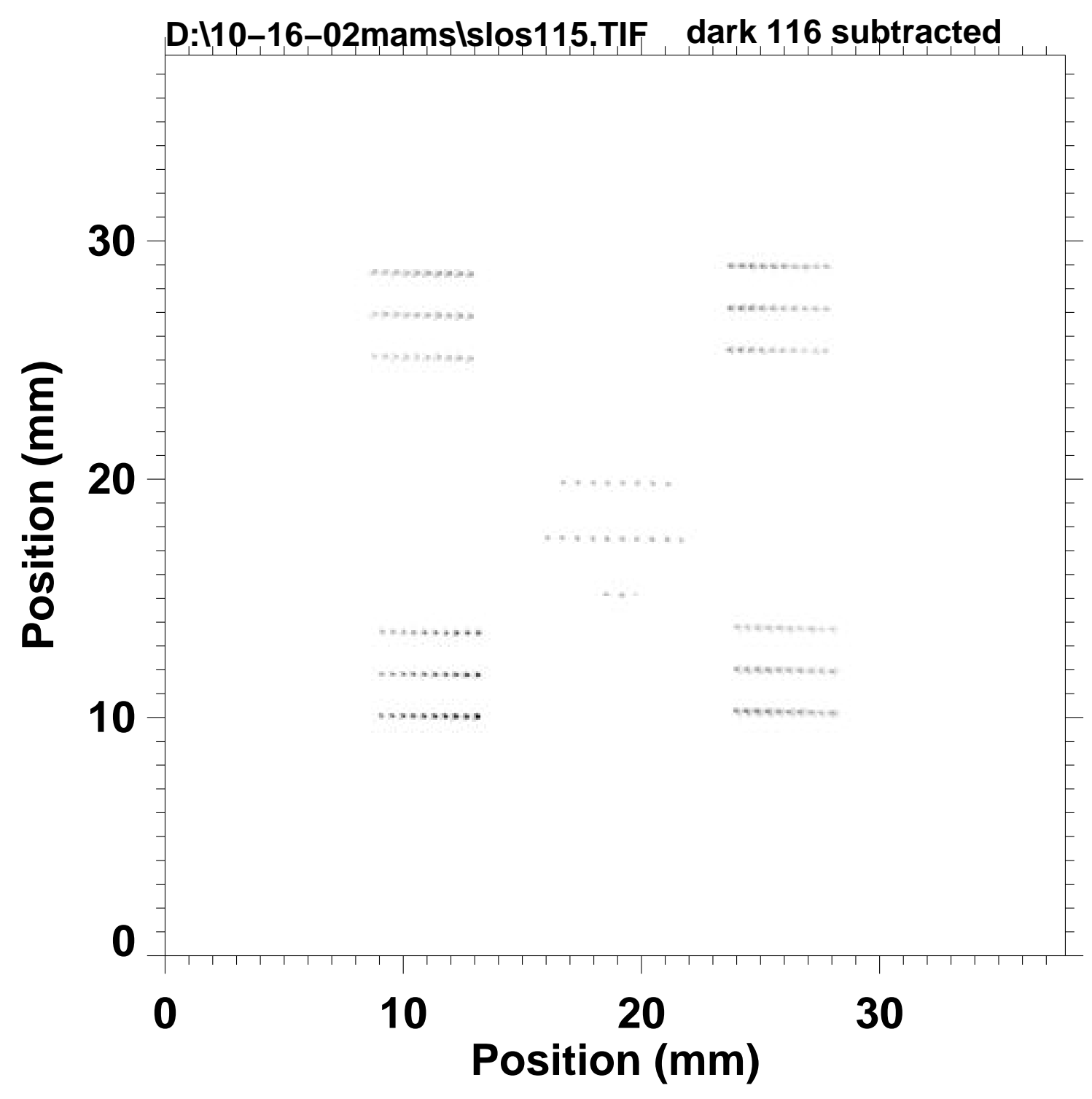

Figure 2: Multi-Anode Manson Source Test of SLOS using improved power supply. A pair of resolution grids was crossed: $75 \mu \mathrm{m}$ slits on $1500 \mu \mathrm{m}$ centers and $100 \mu \mathrm{m}$ slits on $400 \mu \mathrm{m}$ centers. The CCD pixels are $9 \mu \mathrm{m}$. The electron optical magnification was about 1.1 with $9 \mu \mathrm{m}$ pixels. The image was taken with a dc bias only on a "sister plate" (micro-striplines structures absent) MCP. For 
micro-strip operation larger dissector voltages would needed to increase the distance between images.
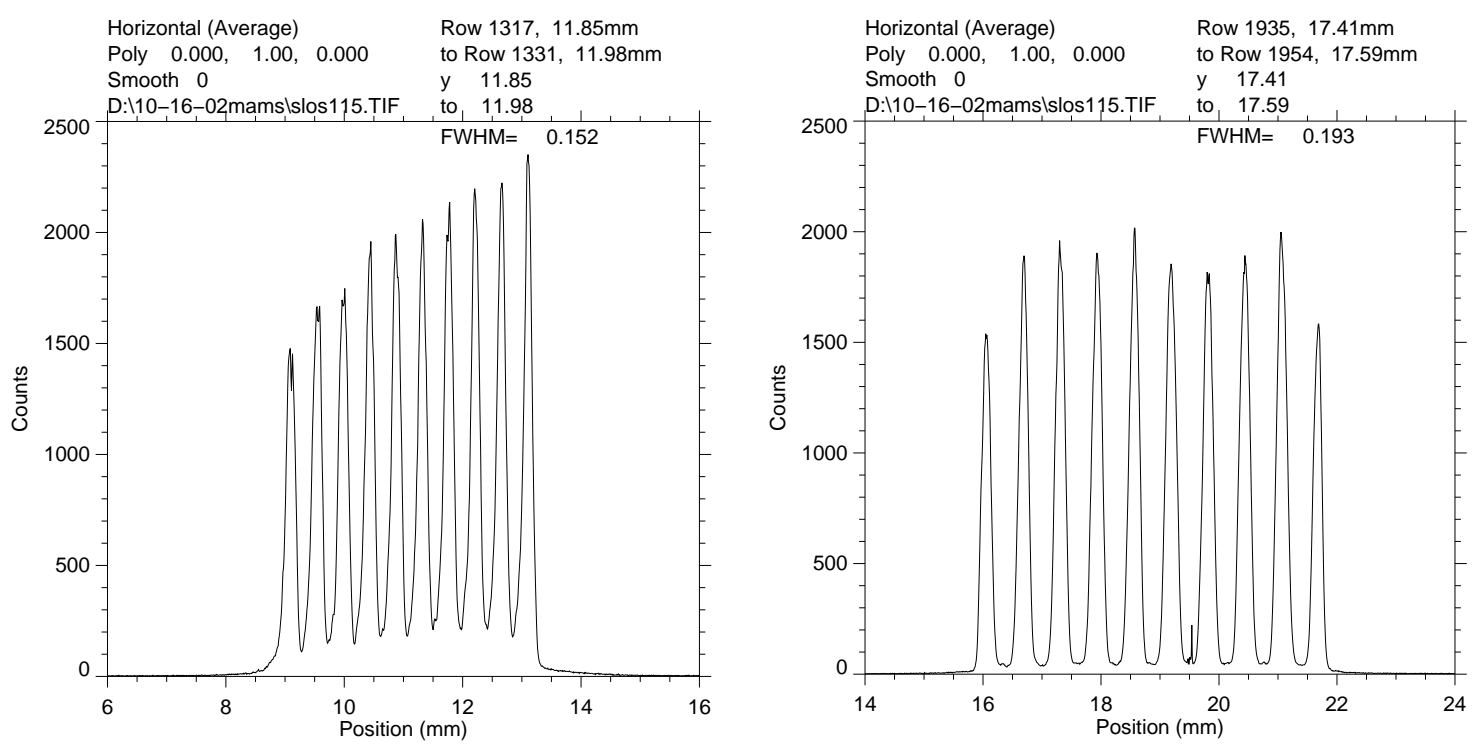

Figure 3: Horizonal Line-outs of Lower Left Dissected (left) and thru X-ray (right) images from Figure 2. For the electron image the slit spacing shows that the electron optics magnification is $\sim 1.11$, so the magnified $100 \mu \mathrm{m}$ slit width is $111 \mu \mathrm{m}$. A Gaussian PSF with a FWHM of $129 \mu \mathrm{m}$ convolved with such an effective slit gives the observed width of $152 \mu \mathrm{m}$. This corresponds to a photocathode point spread function with a FWHM of $116 \mu \mathrm{m}$. The "thru x-ray" image has a point projection magnification of 1.57 due to the size of the electron optics and the distance of the resolution masks to the x-ray source. The observed $193 \mu \mathrm{m}$ line-widths can be modeled by convolving the 1.57 magnified $100 \mu \mathrm{m}$ slit, a FWHM Gaussian MCP PSF of $50 \mu \mathrm{m}$ and the "pinhole camera" 0.57 de-magnified image of the $\sim 250 \mu \mathrm{m}$ FWHM Gaussian source size of the Manson x-ray source. 


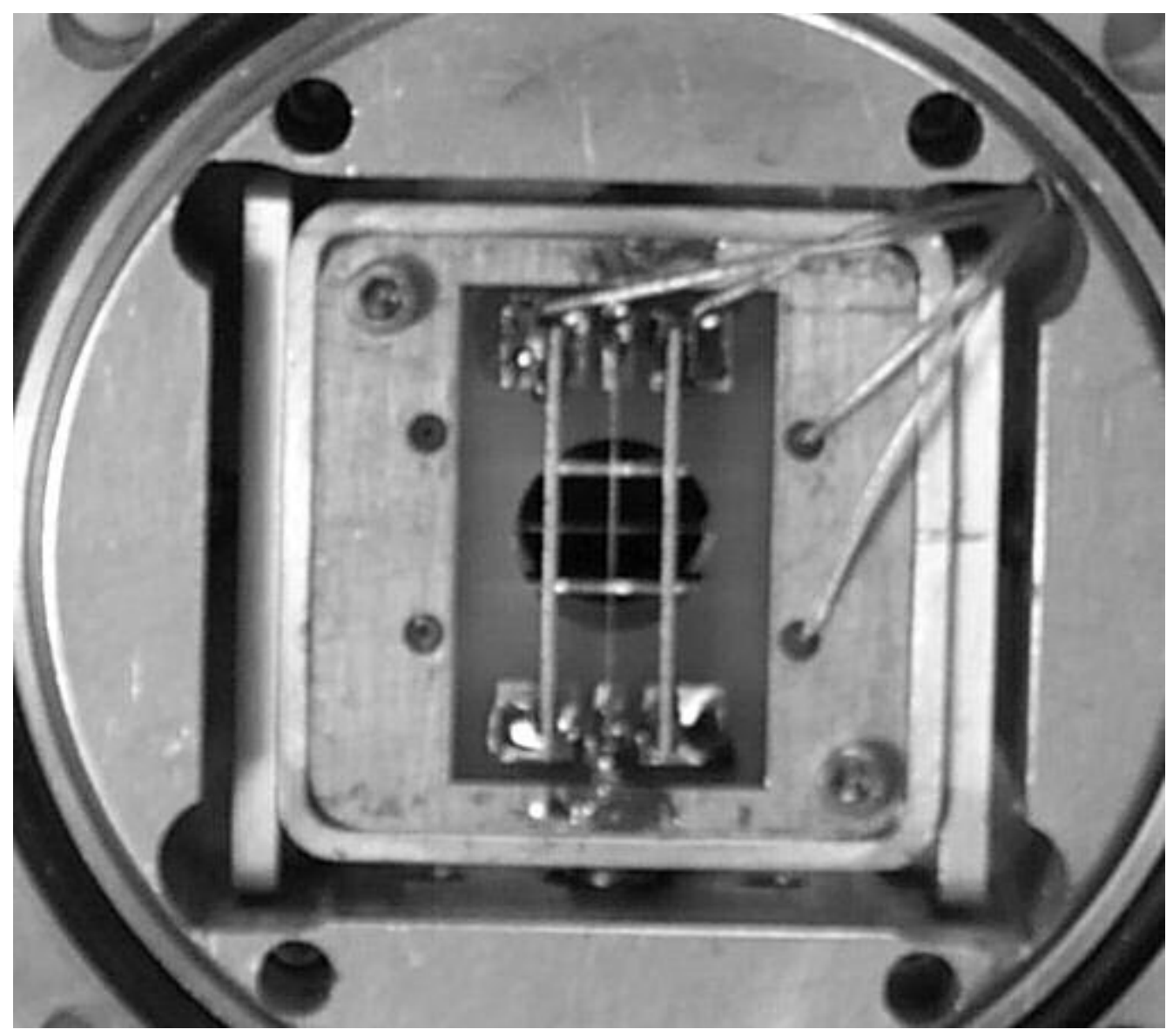

Fi gure 4: Picture of SLOS thick outer plate dissector /dual bi-prism built using improved fixture. 


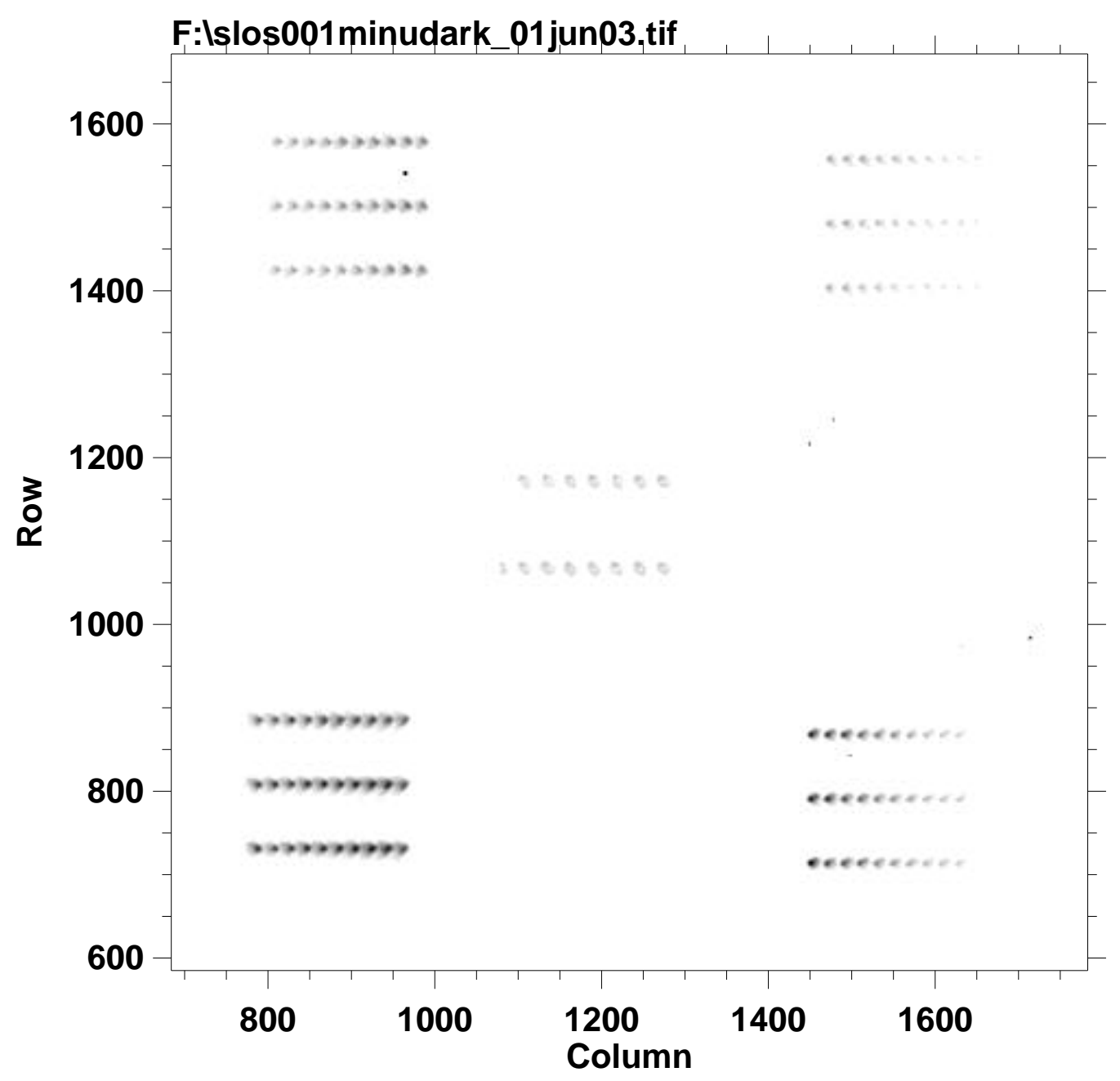

Figure 5: Multi-Anode Manson Source Test of SLOS using improved power supply and thick outer plate dissector /dual bi-prism built using improved fixture. A pair of resolution grids was crossed: $75 \mu \mathrm{m}$ slits on $1500 \mu \mathrm{m}$ centers and $100 \mu \mathrm{m}$ slits on $400 \mu \mathrm{m}$ centers. A lens coupled $2 \mathrm{k}$ by $2 \mathrm{k}$ CCD camera was used. The image was taken with a dc bias only on a "sister plate" (micro-striplines structures absent) MCP. This is best image available of the new configuration. Although the interimage balance is not optimized, the point aberrations of each dissected image are more self-similar between the electron images. The x-ray source was operating at higher current than in the previous figures. The number of "through" $x$-rays un-shadowed by the anode aperture is different since the image tube / source configuration was changed. 


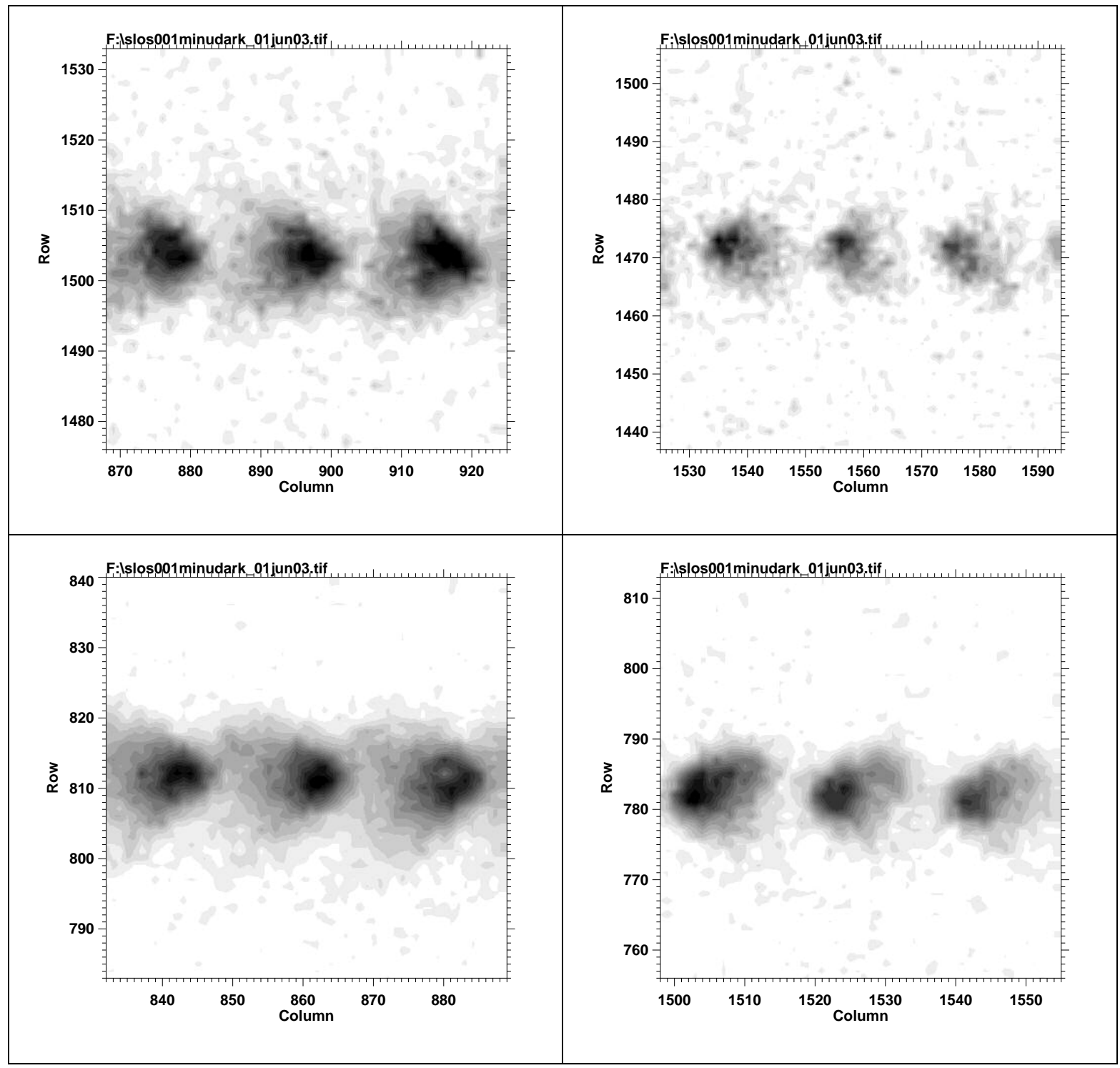

Figure 6 Details of Figure 5. Although the inter-image balance is not optimized, the point aberrations of each dissected image are more self-similar between the electron images. 